\title{
Acquired Angioedema With Anti-C1-inhibitor Autoantibodies During Assisted Reproduction Techniques
}

Marbán Bermejo E ${ }^{1}$, Caballero T ${ }^{2,3}$, López-Trascasa $\mathrm{M}^{4}$, Caballero Peregrín $\mathrm{P}^{1}$, Gil Herrera $\mathrm{J}^{1}$

${ }^{I}$ Clínica Tambre, Madrid, Spain

${ }^{2}$ Servicio de Alergia, Hospital La Paz Health Research Institute (IdiPaz), Madrid, Spain

${ }^{3}$ BiomedicalResearch Network on Rare Diseases (CIBERER, U754), Madrid, Spain

${ }^{4}$ Unidad Inmunologia, Hospital La Paz Health Research Institute (Idipaz), Madrid, Spain

J Investig Allergol Clin Immunol 2018; Vol. 28(1): 62-64 doi: $10.18176 /$ jiaci.0213

Key words: Acquired angioedema. C1-Inhibitor deficiency. Autoimmunity. Assisted reproduction techniques.

Palabras clave: Angioedema adquirido. Déficit de C1-Inhibidor. Autoinmunidad. Técnicas de reproducción asistida.

C1-inhibitor $(\mathrm{Cl}-\mathrm{INH})$ deficiency activates the contact system, resulting in increased bradykinin, vascular hyperpermeability, and recurrent, localized, and self-limiting acute angioedema attacks [1]. Angioedema is classified as hereditary (C1-INH-HAE) or acquired (C1-INH-AAE) [1].

C1-INH-HAE is an autosomal dominant inherited disorder caused by mutations in the $C 1-I N H$ gene, with an estimated prevalence of approximately $1 / 50000$ inhabitants [1]. C1-INHAAE is much less prevalent (around 1/500 000) and is often associated with the presence of anti-C1-INH autoantibodies and/or lymphoproliferative disorders $[1,2]$.

Estrogens and pregnancy can worsen the clinical expression of $\mathrm{C} 1$-INH-HAE [3-5], whereas the fertility rate is similar in patients with C1-INH-HAE and in the general population [3]. However, to our knowledge, there are no previous reports on pregnancy and C1-INH-AAE.

We present the case of a 38-year-old woman who attended our clinic wishing to become pregnant. She underwent 3 intrauterine insemination cycles with sperm from a donor, which were unsuccessful. Five months after having initiated assisted fertilization techniques, she experienced a first episode of nonerythematous, nonpruriginous angioedema affecting her feet. The patient had previously tolerated oral contraceptives containing estrogens (drospirenone/ ethinylestradiol). Thereafter, she underwent ovarian stimulation with recombinant human luteinizing hormone, recombinant human follicle-stimulating hormone, recombinant human chorionic gonadotropin, and micronized natural progesterone for in vitro fertilization (IVF). The embryos were cryopreserved owing to her high progesterone levels. During this period, she experienced several self-limiting acute edema episodes affecting the upper/lower limbs. One of these episodes also affected the glottis and required admission 
to the emergency department, where she was treated with diuretics and corticosteroids, without improvement. An immunological study revealed a very low $\mathrm{C} 4$ serum level $(2.2 \mathrm{mg} / \mathrm{dL})$, normal antigenic C1-INH (16.2 mg/dL), low C1-INH function (25.7\%), and normal serum C1q (157.95 $\mu \mathrm{g} / \mathrm{dL})$. Sequencing of the $C 1-I N H$ and $F 12$ genes revealed no pathogenic variants. She was initially diagnosed with $\mathrm{C} 1-$ INH-HAE and was referred to the angioedema unit of a tertiary hospital, where her diagnosis was changed to C1-INH-AAE because of the presence of anti-C1-INH IgG autoantibodies. An exhaustive study was performed, and lymphoproliferative disorder was excluded. Additional autoimmunity tests showed negative results, and a normal peripheral lymphocyte count with $15 \%$ NK cells was found. After the diagnosis, the patient was prescribed plasma derived $\mathrm{C} 1$-inhibitor concentrate (pdhC1INH) (Berinert, CSL-Behring) to be taken at home. Off-label intravenous pdhC1INH (1000U) was administered for subsequent angioedema episodes, with a good response and no adverse events.

After the failed IVF cycles, the patient underwent egg and sperm donation treatment. One fresh embryo was transferred, resulting in a monochorionic monoamniotic twin pregnancy. She experienced a miscarriage in the eighth week of pregnancy as a complication of the monochorionic monoamniotic twin pregnancy, although this was unrelated to angioedema and pdhC1INH use. Uterine curettage was performed with prior administration of intravenous pdhC1INH $1000 \mathrm{U}$ as short-term prophylaxis, with no subsequent angioedema. In the second cycle, natural cycle endometrial preparation was used to transfer another frozen embryo. A single blastocyst was transferred, leading to pregnancy. Surprisingly, the approach resulted in a monochorionic biamniotic twin pregnancy. Adjuvant therapy included intravenous gammaglobulin (Privigen, CSL-Behring) every 3 weeks because of NK percentages [6] and evidence of autoimmunity [7]; vitamins and ferrous oral supplements were also administered. Autoimmune thyroid dysfunction was first detected at the end of the first trimester, and thyroxin was prescribed. During the second and third trimesters, complement levels returned to normal and no angioedema attacks occurred. The patient underwent cesarean delivery at the 37 th week owing to breech presentation, with prior administration of intravenous pdhC1INH $1000 \mathrm{U}$ as shortterm prophylaxis. No angioedema developed. The patient gave birth to 2 healthy boys weighing $2900 \mathrm{~g}$ and $3100 \mathrm{~g}$ with no further obstetrical or neonatal complications. She started breastfeeding immediately, together with artificial lactation, and is currently ( 21 months after delivery) breastfeeding them twice a day. She remained free of angioedema attacks for 3.5 months after delivery, when she started to experience moderate-severe angioedema episodes at various sites, which were treated with intravenous pdhC1INH 1000U twice a month. She also reported monthly mild abdominal attacks, which resolved spontaneously.

During a short period with frequent angioedema attacks, the patient received long-term prophylaxis with intravenous pdhC1INH every 4 days.

Complement levels, angioedema attacks, and treatments are summarized in the Figure.

We report the case of a patient who developed C1-INHAAE during fertilization therapy. Our initial diagnosis was

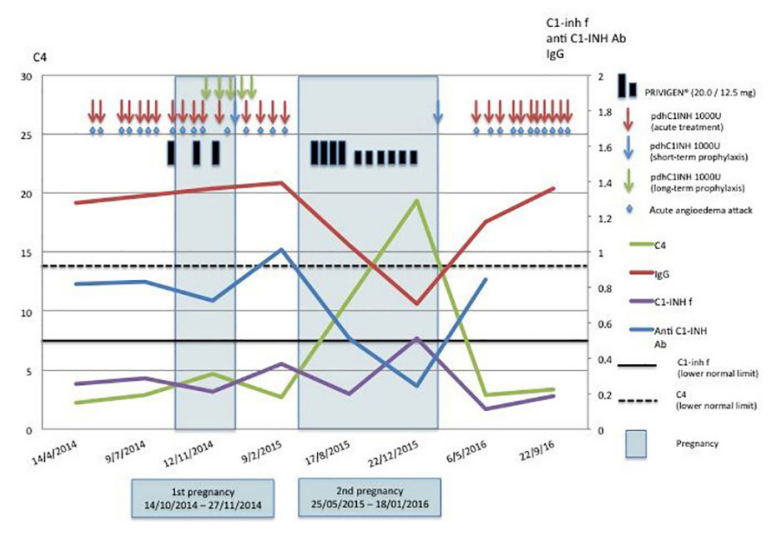

Figure. Clinical and immunological evolution. The patient had a first angioedema (AE) attack 5 months after starting with assisted fertilization treatments. She subsequently had angioedema attacks that coincided with ovarian stimulation for in vitro fertilization and pregnancy. The patient did not have angioedema attacks during the second pregnancy, which coincided with administration of intravenous immunoglobulin (Privigen), a decrease in total IgG and anti-C1-INH antibodies and an increase in C1-INH function and C4. C4, mg/dL (normal range, 14-60); $\mathrm{lgG}, \mathrm{mg} / \mathrm{dL}$ (normal range, 725-1900); C1-INH level, mg/dL (normal range, 16-33); C 1-INH function, \% (normal range, >50).

C1-INH-HAE, since C1q was normal, a mutation in the C1INH gene is not found in $5 \%-10 \%$ of families with $\mathrm{C} 1-\mathrm{INH}-$ HAE [4], and up to $25 \%$ of patients can have a spontaneous mutation $[1,4]$. Subsequently, positive anti-C1-INH antibodies enabled us to make a more accurate diagnosis of C1-INHAAE. A case of C1-INH-AAE with normal $\mathrm{Clq}$ has been reported [8].

Patients with C1-INH-HAE or C1-INH-AAE can attend a fertility clinic if they cannot become pregnant naturally. They should avoid estrogen treatments, which can lead to an increase in the frequency and severity of angioedema attacks [4]. Therefore, if ovarian stimulation is necessary for IVF, it should be targeted to reach the lower estrogen levels [4]. Besides, pdhC1INH can be used during these procedures in order to reduce the number and severity of angioedema attacks [4]. pdhC1INH is the first-line treatment for $\mathrm{C} 1$ INH-HAE during pregnancy [4] and should also be used for C1-INH-AAE. In the case we report, adjuvant therapy with intravenous gammaglobulin may have helped to modulate autoimmune disease, as previously published [6,7], with a reduction in antiC1-INH antibody levels, normalization of $\mathrm{C} 1-\mathrm{INH}$ levels and function and C4 levels, and improvement in clinical symptoms.

Also intriguing was the presentation of 2 consecutive monochorionic twin pregnancies after single-embryo transfers. The monochorionic twin pregnancy rate increases by $1 \%-5 \%$ in single-embryo transfer [9], probably owing to the culture medium, the length of the blastocyst stage, and embryo quality. In a large historical cohort study, where more than 238000 pregnancies after assisted reproduction techniques were analyzed, only 1 woman had recurrence of monochorionic twin pregnancy, although this was considered to be random [9].

The clinical case here described is unique because $\mathrm{C} 1-\mathrm{INH}$ AAE, an extremely rare condition, developed during assisted 
reproduction and was difficult to diagnose because the patient had normal C1q levels despite having anti-C1-INH antibodies.

\section{Acknowledgments}

The authors wish to thank Dr Ana Garrido Quijano (Clínica Tambre, Madrid, Spain), Dr Pedro Gargantilla Madera (Servicio de Medicina Interna, Hospital El Escorial, Madrid, Spain), Dr Ana Ma Lacalle (Servicio de Ginecología y Obstetricia, Hospital Universitario La Paz, Madrid, Spain), and Alberto López-Lera (CIBERER, U754. Unidad de Inmunología. Hospital La Paz). Margarita López-Trascasa received a grant from Instituto de Salud Carlos III and Fondos Feder (PI15-00255).

\section{Funding}

The authors declare that no funding was received for the present study.

\section{Conflicts of Interest}

Teresa Caballero has received speaker/consultancy fees and funding for travel/meetings and has participated in clinical trials for ByoCryst, CSL Behring, GlaxoSmithKline, MSD, Novartis, Pharming NV, Shire, and Sobi. She is a researcher from the IdiPaz program for promoting research activities.

Esther Marbán Bermejo, Margarita López-Trascasa, and Juana Gil Herrera declare that they have no conflicts of interest.

\section{References}

1. Cicardi M, Aberer W, Banerji A, Bas M, Bernstein JA, Bork K, et al. Classification, Diagnosis And Approach To Treatment Of Angioedema: Consensus Report From The Hereditary Angioedema International Working Group. Allergy. 2014;69:602-16.

2. Zanichelli A, Azin GM, Wu MA, Suffritti C, Maggioni L, Caccia $S$, et al. Diagnosis, Course, and Management of Angioedema in Patients With Acquired C1-Inhibitor Deficiency. J Allergy Clin Immunol Pract. 2017:5:1307-13.

3. Bouillet L, Longhurst $H$, Boccon-Gibod I, Bork K, Bucher C, Bygum $A$, et al. Disease expression in women with hereditary angioedema. Am J Obstet Gynecol. 2008;199:484.e1-4. Epub 2008 Jun 13.

4. Caballero T, Farkas H, Bouillet L, Bowen T, Gompel A, Fagerberg $C$, et al. International consensus and practical guidelines on the gynecologic and obstetric management of female patients with hereditary angioedema caused by $\mathrm{C} 1$ inhibitor deficiency. J Allergy Clin Immunol. 2012 Feb;129(2):308-20.

5. González-Quevedo T, Larco Jl, Marcos C, Guilarte M, Baeza ML, Cimbollek S, et al. Management of Pregnancy and Delivery in Patients With Hereditary Angioedema Due to C1 Inhibitor Deficiency. J Investig Allergol Clin Immunol. 2016;26(3):161-7.

6. Ramos-Medina R, García-Segovia A, Gil J, Carbone J, Aguarón de la Cruz A, Seyfferth A, et al. Experience in IVIg therapy for selected women with recurrent reproductive failure and NK cell expansion. Am J Reprod Immunol. 2014;71:458-66.

7. Sánchez-Ramón S, Comins-Boo A, Núñez Beltrán M, García Segovia Á, Gil Herrera J. The absence of evidence is not the evidence of absence. Hum Reprod. 2016;31:217-8.
8. Bova M, Petraroli A, Loffredo S, Marone G, Triggiani M. A Unique Case of Angioedema With Anti-C1 Inhibitor Antibodies and Normal C 1 Inhibitor Levels. J Investig Allergol Clin Immunol. 2016;26(2):111-2.

9. Luke B, Brown MB, Wantman E, Stern JE. Factors associated with monozygosity in assisted reproductive technology pregnancies and the risk of recurrence using linked cycles. Fertil Steril. 2014;101:683.

Manuscript received July 10, 2017; accepted for publication November 14, 2017.

Esther Marbán Bermejo Clínica Tambre C/. Tambre, 8 28002 Madrid, Spain E-mail:emarban@clinicatambre.com 